\title{
Atividade antioxidante e compostos bioativos em espécies de um fragmento de Cerrado goiano tipo cerradão
}

Antonio Carlos Pereira de Menezes Filho, Josemar Gonçalves Oliveira Filho, Marcela Christofoli, Carlos Frederico de Souza Castro

Instituto Federal de Educação, Ciência e Tecnologia Goiano - IF Goiano. Universidade Estadual Paulista - UNESP. Universidade Federal de Goiás - UFGO. E-mail: astronomoamadorgoias@gmail.com

\section{Resumo}

O Cerrado apresenta uma exuberante flora adaptada aos mais variados tipos fitofisionômicos, onde o cerradão apresenta inúmeras espécies que constituem a flora brasileira. $O$ objetivo deste estudo foi avaliar os teores de compostos fenólicos, atividade antioxidante, $\beta$-caroteno, licopeno e provitamina $A$ em extratos etanólicos foliares de dezessete espécies vegetais coletadas em um fragmento de Cerrado no município de Rio Verde - GO, caracterizado como cerradão. Os resultados apresentaram expressivos teores de fenólicos totais e atividade antioxidante, exceto para $A$. humile, $B$. intermedia e $K$. lathrophytum que não houve atividade antioxidante, bem como quantidades significativas de betacaroteno e provitamina $A$, para algumas delas. Foram observados também altos teores de licopeno em todos os extratos foliares avaliados. As espécies vegetais do Cerrado caracterizam importante material fitoquímico para produção de medicamentos e alimentos ricos em compostos químicos importantes para a saúde e bem estar humano e animal.

Palavras-chave: DPPH; extratos foliares; $\beta$-caroteno.

\author{
Antioxidant activity and bioactive compounds in species of a fragment of Goiano the Cerrado type \\ cerradão
}

\begin{abstract}
The Cerrado presents an exuberant flora adapted to the most varied types of phytophysiognomies, where the cerradão presentes numerous species that constitute the Brazilian flora. The objective of this study was to evaluate the levels of phenolic compounds, antioxidant activity, $\beta$-carotene, lycopene and provitamin $A$ in foliar ethanolic extracts of seventeen plant species collected in a Cerrado fragmente in the municipality of Rio Verde - GO, characterized as cerradão. The results presented significant levels of total phenolics and antioxidant activity, except for $A$. humile, $B$. intermedia and $K$. lathrophytum that there was no antioxidant activity, as well as significant amounts of beta-carotene and provitamin A, for some of them. High levels of lycopene were also observed in all leaf extracts evaluated. The Cerrado plant species characterize important phytochemical material for the production of drugs and foods rich in chemical compounds important for human and animal health and welfare.
\end{abstract}

Keywords: DPPH; leaves extracts; $\beta$-carotene.

\section{Introdução}

O conhecimento fitoterápico é usado há milênios para o tratamento de doenças, simbolizando muitas vezes a única forma de tratamento, visto que, os produtos medicamentosos sintetizados custam caro, para a maioria da população (BURQUE et al., 2015; SILVA et al., 2013).

Atualmente as indústrias farmacêuticas e laboratórios de pesquisa vêm pesquisando 
compostos fitoterápicos com atividade antioxidante, presentes em inúmeras espécies vegetais da flora brasileira. As plantas produzem metabólitos secundários constantemente e em quantidade variáveis, durante as duas sazonalidades (seca e chuvosa) (BURQUE et al., 2015). De acordo com Halliwell et al. (1995), os metabólitos secundários apresentam eficácia como agentes redutores, no sequestro de radicais livres como o oxigênio singleto e alguns íons metálicos. Alguns compostos podem agir simultaneamente em mais de uma função, aumentando assim, o efeito benéfico que os mesmos promovem.

Diversas espécies vegetais endêmicas do Cerrado ainda não possuem estudos físicoquímicos e fitoquímicos quanto aos seus possíveis compostos bioativos, sendo necessárias novas pesquisas sobre estas espécies para constituição de um banco farmacológico abundante, contribuindo para as pesquisas quanto a possível utilização destes fitocompostos nas áreas da saúde, alimentícia, dentre outras.

Os vegetais produzem naturalmente compostos de primeira e segunda ordem como metabólitos, à quantidade destes compostos varia devido a agentes intrínsecos e extrínsecos. Vários destes compostos fitoquímicos apresentam atividade antioxidante sequestrando radicais livres produzidos ou adquiridos pelos seres humanos e também em alimentos. Os compostos fenólicos enquadram também como agentes antioxidantes participando diversas categorias de compostos, como os fenóis simples, ácidos fenólicos, cumarinas, flavonóides e estilbenos (SOUSA et al., 2007; NACZK; SHAHIDI, 2004). Os agentes antioxidantes possuem função de neutralização ou sequestro de radicais livres, como o oxigênio singleto e na quelação de metais de transição, agindo em duas etapas, de iniciação e na propagação do processo oxidativo (SOARES, 2002).

Outros compostos como os carotenóides ( $\beta$-caroteno e licopeno) agem como agentes antioxidantes protegendo os lipídios dos danos causados pela peroxidação e do oxigênio singleto. O sistema de ação desencadeado pelos carotenóides é de deslocação dos elétrons desemparelhados através da estrutura de ligações duplas conjugadas (SOUSA et al., 2007; VALKO et al., 2004). A provitamina A é um termo genérico para os compostos, retinol e seus ésteres. A sua presença nos vegetais pode variar quantitativamente entre os mais variados grupos.
Sendo também incluída nos grupos dos compostos com característica antioxidante. Além disso, a hipovitaminose é uma doença crônica ocasionada pela falta desta vitamina no organismo podendo acarretar problemas sérios como a síndrome ocular, a xeroftalmia e a cegueira irreversível (SOUZA; BOAS, 2002; BENDICH; LANGSETH, 1989; RONCADA et al., 1978).

Algumas das espécies como o cajuzinhodo-campo (Anacardium humile) vêm sendo estudado demonstrando que a planta apresenta atividade antifúngica, anti-inflamatória, antitumoral e inseticida natural (PEREIRA et al., 2018; LUIZ-FERREIRA et al., 2008), Byrsonima sp., conhecida popularmente por murici, são utilizadas como plantas fitoterápicas, para o tratamento de infecções gastrointestinais, apresentando ações anti-inflamatórias, antimicrobiana e antioxidante (PEREIRA et al., 2015; MALDINI et al., 2009; MICHELIN et al., 2008), Solanum lycocarpum utilizada também como medicamento fitoterápico com atividade diurética, calmante, antiespasmódica, antiofídica, infecções do trato urinário, cólicas abdominais e renais, e antiepiléptica (GALLON et al., 2015).

O objetivo deste trabalho foi verificar atividade sequestradora (atividade antioxidante), conteúdos de fenólicos totais, teores de carotenóides ( $\beta$-caroteno e licopeno) e provitamina A em dezessete extratos foliares de espécies encontradas em um fragmento de Cerrado tipo cerradão no município de Rio Verde - Goiás.

\section{Material e Métodos}

As folhas de Anacardium humile, Annona crassiflora, Brosimum gaudichaudii, Byrsonima coccolobifolia, Byrsonima intermedia, Cardiopetalum calophyllum, Campomanesia adamantium, Curatella americana, Dimorphandra mollis, Hymenaea stigonocarpa, Kielmeyera lathrophytum, Qualea grandiflora, Rollinia laurifolia, Salvertia convallariodora, Solanum cernuum, Solanum lycocarpum e Terminalia argentea, foram coletadas nas primeiras horas da manhã, no mês de julho/2018, sendo armazenadas em embalagens de polietileno de cor preta. A identificação de todas as espécies vegetais foi realizada pelo primeiro autor deste estudo.

As coletas foram realizadas em uma área de preservação permanente (APP) localizada no município de Rio Verde-GO, pertencente à 
Universidade de Rio Verde-GO, com as seguintes coordenadas geográficas: $17^{\circ} 47^{\prime} 11.3^{\prime \prime} \mathrm{S}$ $50^{\circ} 57^{\prime} 59.0^{\prime \prime} \mathrm{W}$. Logo após, as amostras foram levadas para o Laboratório de Produtos Naturais no Instituto Federal Goiano, Campus Rio Verde$\mathrm{GO}$, onde foram lavadas em água corrente e deixadas para secar sob folhas de papel toalha para retirada do excesso de água.

Para o preparo do extrato, $100 \mathrm{~g}$ de folhas secas foram moídas em moinho de facas tipo ciclone obtendo-se um pó fino. A maceração foi realizada pela extração em $100 \mathrm{~mL}$ de etanol e em acetato de etila na proporção (1:1), por 7 dias, em temperatura ambiente. Logo após, os extratos foram filtrados em papel filtro. As alíquotas dos extratos etanólicos e de acetato de etila foram rotaevaporados sob pressão reduzida a $50 \pm 1,0{ }^{\circ} \mathrm{C}$ em rotaevaporador rotativo, obtendo-se os extratos brutos concentrados. Em seguida os extratos brutos foram dissolvidos em solução etílica $66 \%$, para serem utilizados nos ensaios de atividade antioxidante, conteúdo de fenóis totais e carotenóides; conforme descrito por Nunes; Dias e Cavalcante (2016) modificado.

A atividade antioxidante proposta para este estudo seguiu metodologia descrita por Rocha et al. (2013) com modificações. A partir dos extratos brutos, acetoetanólicos, foram realizadas suspensões com $15 \mathrm{~mL}$ de etanol $66 \%$. Em cada tubo de ensaio foi adicionada $0,5 \mathrm{~mL}$ de cada extrato acrescido com $1,5 \mathrm{~mL}$ de solução de radical livre 2,2-difenil-1-picril-hidrazil (DPPH).

Logo após, os tubos foram homogeneizados em Vortex por 5 segundos e deixados em local ao abrigo de luz e calor por 30 minutos após o início da reação de remoção. As leituras foram realizadas no comprimento de ondas de $517 \mathrm{~nm}$ em espectrofotômetro UV-Vis. A solução controle foi constituída de etanol $95 \%$ P.A. e Acetato de Etila 99\% P.A. Os resultados foram expressos em \% de remoção conforme equação 1 :

$\%$ Remoção $=\left(\frac{\text { Abs controle }- \text { Abs extrato }}{\text { Abs controle }}\right) * 100$

Eq.1

A porcentagem de remoção de DPPH, foi determinada através de gráfico com os resultados obtidos, lançando-se os valores de concentração em $\mathrm{mg} \mathrm{L}^{-1}$ como abcissas e as porcentagens de remoção dos extratos como coordenadas. A reta foi determinada para o cálculo de $\mathrm{EC}_{50}$ (quantidade de extrato necessário para reduzir $50 \%$ a concentração inicial de DPPH), conforme recomendação por Rocha et al. (2013) e desenvolvida por Blois (1958).

O conteúdo de compostos fenólicos totais foi determinado conforme descrito por Nunes; Dias e Cavalcante (2016) modificado. Em um tubo de ensaio uma alíquota de $0,5 \mathrm{~mL}$ da solução de extrato etanólico $66 \%$, foi acrescida com $8 \mathrm{~mL}$ de água deionizada e $0,5 \mathrm{~mL}$ de uma solução de reagente de Folin-Ciocalteau na proporção (1:9) em água deionizada. A amostra foi homogeneizada em Vortex por 10 segundos e acrescida com $1 \mathrm{~mL}$ de solução saturada de carbonato de sódio $7,5 \%(\mathrm{~m} / \mathrm{m})$ e novamente homogeneizada. A amostra foi deixada em repouso ao abrigo da luz e calor por 60 minutos após início da reação. As leituras foram realizadas em espectrofotômetro UV-Vis no comprimento de onda de $720 \mathrm{~nm}$. Foi montada uma curva padrão de ácido gálico nas concentrações entre 40 a $400 \mathrm{mg} / \mathrm{L}^{-1}$. Para o branco, foi utilizada água deionizada.

A partir da curva padrão, realizou-se o cálculo do conteúdo de compostos fenólicos totais, expressos em $\mathrm{mg}$ equivalente em ácido gálico (EAG) por $100 \mathrm{~g}^{-1} \mathrm{MS}$.

A quantificação dos teores de $\beta$-caroteno e licopeno foi realizada conforme descrito por Nagata e Yamashita (1992) com modificações. Utilizou-se $1 \mathrm{~mL}$ da solução de extrato etanólico $66 \%$. As leituras foram realizadas nos seguintes comprimentos de ondas: 453, 505, 645 e $663 \mathrm{~nm}$. Para determinação, foi utilizado as seguintes equações, 2 e 3 . Inicialmente os resultados foram expressos em mg $100 \mathrm{~mL}^{-1}$, logo após, os resultados foram multiplicados por 1.000 , sendo expressos em $\mu \mathrm{g} 100 \mathrm{~g}^{-1}$ de massa seca.

$\beta$-caroteno $=\left(0,216 \times A_{663}\right)-\left(1,22 \times A_{645}\right)-(0,304$
$\left.\times A_{505}\right)+\left(0,452 \times A_{453}\right)$
Licopeno $=\left(0,0458 \times A_{663}\right)+\left(0,204 \times A_{645}\right)+(0,372$
$\left.\times A_{505}\right)-\left(0,0806 \times A_{453}\right)$

Para equivalente de retinol (vitamina $A$ ), adotou-se a razão de conversão de $12 \mu \mathrm{g} \beta$ caroteno, que corresponde a 1 RAE (Retinol Activity Equivalent), conforme descrito por Ferreira et al. (2008).

Os experimentos foram realizados em triplicata e os resultados médios foram comparados através do teste de Tukey a $5 \%$ de significância, utilizando o programa Software PAST3. Os resultados foram apresentados pela média \pm desvio padrão. 


\section{Resultados}

$\mathrm{Na}$ Tabela 1 são apresentados os resultados para compostos fenólicos totais e atividade antioxidante das 17 espécies vegetais coletadas.

Tabela 1. Compostos fenólicos totais e atividade antioxidante por remoção do radical DPPH em extratos vegetais foliares de espécies encontradas em um fragmento de Cerrado tipo cerradão, no município de Rio Verde - Goiás.

\begin{tabular}{|c|c|c|}
\hline Extratos & $\begin{array}{l}\text { Compostos fenólicos totais } \\
\left.\text { (mg EAG } 100 \mathrm{~g}^{-1} \mathrm{MS}\right)\end{array}$ & $\begin{array}{c}\text { Remoção de } \\
\text { DPPH }\end{array}$ \\
\hline Anacardium humile & $12,39 \pm 0,09^{d}$ & $\mathrm{nd}^{* \mathrm{a}}$ \\
\hline Annona crassiflora & $10,48 \pm 0,15^{\mathrm{ef}}$ & $44,87 \pm 0,30^{d}$ \\
\hline Brosimum gaudichaudii & $13,04 \pm 0,44^{d}$ & $67,72 \pm 0,24^{\mathrm{g}}$ \\
\hline Byrsonima coccolobifolia & $6,40 \pm 0,03^{i}$ & $76,01 \pm 0,36^{h}$ \\
\hline Byrsonima intermedia & $11,18 \pm 0,10^{\mathrm{e}}$ & $n d^{* b}$ \\
\hline Cardiopetalum calophyllum & $14,90 \pm 0,12^{c}$ & $68,16 \pm 0,12^{g}$ \\
\hline Campomanesia adamantium & $10,73 \pm 0,18^{e}$ & $40,47 \pm 0,60^{c}$ \\
\hline Curatella americana & $9,77 \pm 0,05^{\mathrm{fg}}$ & $45,85 \pm 0,67^{d}$ \\
\hline Dimorphandra mollis & $7,51 \pm 0,03^{h}$ & $65,60 \pm 0,56^{g}$ \\
\hline Hymenaea stigonocarpa & $20,21 \pm 0,24^{b}$ & $49,85 \pm 0,37^{e}$ \\
\hline Kielmeyera lathrophytum & $23,82 \pm 0,07^{a}$ & $n d^{* a}$ \\
\hline Qualea grandiflora & $15,31 \pm 0,45^{c}$ & $57,35 \pm 0,55^{f}$ \\
\hline Rollinia laurifolia & $23,05 \pm 0,08^{a}$ & $68,02 \pm 0,32^{\mathrm{g}}$ \\
\hline Salvertia convallariodora & $9,23 \pm 0,04^{g}$ & $49,60 \pm 0,37^{e}$ \\
\hline Solanum cernuum & $9,48 \pm 0,07^{g}$ & $66,83 \pm 0,36^{g}$ \\
\hline Solanum lycocarpum & $14,94 \pm 0,28^{c}$ & $67,08 \pm 0,18^{g}$ \\
\hline Terminalia argentea & $11,12 \pm 0,08^{\mathrm{e}}$ & $43,98 \pm 2,36^{d}$ \\
\hline
\end{tabular}

mg EAG $100 \mathrm{~g}^{-1}$ MS= equivalentes em ácido gálico. (\%) de remoção de DPPH = radical [2,2-difenil-1-picril-hidrazil]. nd* = Não detectado. Médias comparadas na mesma coluna, seguidas de mesma letra não diferem significativamente pelo teste de Tukey a $(p<0,05)$.

Os compostos fenólicos totais apresentaram médias variadas, com maiores teores verificadas nos extratos de $K$. lathrophytum, R. laurifolia, H. stigonocarpa. Os menores teores foram registrados para os extratos etanólicos foliares de $B$. coccolobifolia, D. mollis, S. convallariodora, S. cernuum, C. americana respectivamente.

Almeida et al. (2014) avaliaram o conteúdo de fenólicos em extratos foliares de Pereskia aculeata e Pereskia grandifolia onde obtiveram teores de 19,34 e 19,17 mg de GAE $100 \mathrm{~g}^{-1}$ respectivamente. Estas espécies são consumidas como alimentos, apresentando teores de mucilagem importantes, bem como de proteínas, sendo utilizadas no preparo de saladas, farinhas, tortas e macarrão (ALMEIDA et al., 2014). Para Andrade et al. (2007) onde foram avaliados o extrato etanólico e frações de diclorometano e de acetato de etila foliar de Acacia podalyriifolia foram encontrados teores de fenóis totais de 206,4; 240,2 e $338,5 \mathrm{mg}$ de GAE $100 \mathrm{~g}^{-1}$. Os autores ainda complementam sobre as inúmeras atividades nos tratamentos de doenças, bem como considerável atividade antioxidante (WU et al., 2005; NAIK et al., 2003).

O conteúdo de fenólicos totais tem recebido especial atenção por apresentarem compostos com atividade antioxidante na captura do oxigênio singleto. Os compostos fenólicos estão envolvidos nas propriedades de óxido-redução, na adsorção e ou neutralização de radicais livres (MENEZES FILHO et al., 2018).

Os resultados da avaliação quantitativa da porcentagem de remoção de DPPH demonstraram que todos os extratos vegetais apresentaram taxa de remoção do radical (\% DPPH), exceto para as espécies $A$. humile, $B$. intermedia e $K$. lathrophytum. Os extratos etanólicos de B. coccolobifolia, C. calophyllum, $R$. laurifólia, B. gaudichaudii, S. lycocarpum, S. cernuum e $D$. mollis apresentaram as maiores porcentagem de atividade antioxidante respectivamente (Tabela 1 ).

Menezes Filho et al. (2018) avaliaram os extratos foliares etanólicos das espécies $B$. coccolobifolia, D. mollis, C. calophyllum, $H$. stigonocarpa e S. lycocarpum coletadas em 
diferente época do ano em relação a este estudo, onde obtiveram valores muito superiores de atividade antioxidante em relação ao presente estudo $(87,56 ; 84,57 ; 84,91 ; 65,68$ e $83,31 \%$ respectivamente). Podemos sugerir que a sazonalidade influencia diretamente nos teores de metabólitos secundários e consequentemente a atividade antioxidante nos vegetais. Nascimento et al. (2011) compararam o consumo de DPPH em extrato metanólico das folhas de Buhinia variegata L. coletadas durante 6 meses do ano e obtiveram atividade antioxidante variando entre 6,84 a $98,32 \%$, sendo que os maiores valores foram observados em amostras coletadas entre os meses de abril, junho, agosto, outubro e dezembro.

Já Burque et al. (2015) obtiveram porcentagem de remoção de DPPH no extrato foliar hidroalcoólico de Lafoensia pacari entre 22,90 a $93,20 \%$. Trabalho desenvolvido por Sousa et al. (2007) encontraram taxa de remoção entorno de $80,0 \%$ no extrato etanólico das folhas de Terminalia brasiliensis.

$\mathrm{Na}$ Tabela 2 estão apresentados resultados para teores de betacaroteno, licopeno e provitamina A obtidos a partir dos extratos etanólicos foliares.

Tabela 2. Teores de $\beta$-caroteno, licopeno e RAE - Retinol activity Equivalent (provitamina A) em extratos vegetais foliares de espécies em um fragmento de Cerrado tipo cerradão no município de Rio Verde - Goiás.

\begin{tabular}{|c|c|c|c|}
\hline Extratos & $\begin{array}{l}\beta \text {-caroteno } \\
\left(\mu \mathrm{g} 100 \mathrm{~g}^{-1}\right)\end{array}$ & $\begin{array}{l}\text { Licopeno } \\
\left(\mu \mathrm{g} 100 \mathrm{~g}^{-1}\right)\end{array}$ & $\begin{array}{l}\text { Provitamina A } \\
\left(\text { RAE } 100 \mathrm{~g}^{-1 *} \text { ) }\right.\end{array}$ \\
\hline Anacardium humile & $\mathrm{nd}^{*}$ & $1551,33 \pm 0,01^{\mathrm{a}}$ & $\mathrm{nd}^{*}$ \\
\hline Annona crassiflora & nd* & $1097,67 \pm 0,00^{\mathrm{e}}$ & nd* \\
\hline Brosimum gaudichaudii & nd* & $698,70 \pm 0,02^{h}$ & nd* \\
\hline Byrsonima coccolobifolia & $544,33 \pm 0,02^{a}$ & $201,67 \pm 0,02$ & $45,36 \pm 1,76^{\mathrm{a}}$ \\
\hline Byrsonima intermedia & $n d^{*}$ & $1468,67 \pm 0,00^{b}$ & nd* \\
\hline Cardiopetalum calophyllum & nd* & $590,67 \pm 0,00^{j}$ & $n d^{*}$ \\
\hline Campomanesia adamantium & nd* & $952,00 \pm 0,00^{f}$ & nd* \\
\hline Curatella americana & nd* & $1072,33 \pm 0,01^{\mathrm{e}}$ & nd* \\
\hline Dimorphandra mollis & $195,33 \pm 0,02^{b}$ & $556,33 \pm 0,01^{k}$ & $16,28 \pm 1,64^{b}$ \\
\hline Hymenaea stigonocarpa & nd* & $1124,33 \pm 0,01^{d}$ & $n d^{*}$ \\
\hline Kielmeyera lathrophytum & nd* & $1339,67 \pm 0,00^{c}$ & nd* \\
\hline Qualea grandiflora & $171,67 \pm 0,01^{c}$ & $645,67 \pm 0,00^{i}$ & $14,31 \pm 0,92^{b}$ \\
\hline Rollinia laurifolia & nd* & $939,00 \pm 0,01^{\mathrm{jk}}$ & nd* \\
\hline Salvertia convallariodora & nd* & $1133,33 \pm 0,00^{d}$ & nd* \\
\hline Solanum cernuum & $26,68 \pm 0,01^{d}$ & $568,67 \pm 0,00^{f}$ & $2,21 \pm 1,03^{c}$ \\
\hline Solanum lycocarpum & nd* & $631,33 \pm 0,01^{i}$ & $\mathrm{nd}^{*}$ \\
\hline Terminalia argentea & nd* & $885,33 \pm 0,00^{\mathrm{g}}$ & nd* \\
\hline
\end{tabular}

RAE $^{*}=$ Retinol activity Equivalent, onde $1 \mathrm{RAE}=1 \mu \mathrm{g}$ de retinol $=12 \mu \mathrm{g} \beta$-caroteno. $\mathrm{nd}^{*}=$ Não detectado. Médias comparadas na mesma coluna, seguidas de mesma letra não diferem significativamente pelo teste de Tukey a $(p<$ $0,05)$.

O $\beta$-caroteno e o licopeno são compostos com atividade antioxidante, devido ao grande número de ligações dieno conjugadas, que em contato com o oxigênio singleto absorvem esse radical livre envolvido em alguns tipos de cânceres e no envelhecimento precoce (MENEZES FILHO et al., 2018; SILVA et al., 2010; BLUM et al., 2005).

Os extratos vegetais avaliados neste estudo apresentaram teores de betacaroteno apenas nas espécies $B$. coccolobifolia, $D$. mollis, $Q$. grandiflora e $S$. cernuum. O carotenóide licopeno foi encontrado em todas as espécies analisadas e os maiores teores ocorreram para as espécies $H$. humile, $B$. intermedia e $K$. lathrophytum (Tabela 2).

Menezes Filho et al. (2018) encontraram teores de $\beta$-caroteno nos extratos etanólicos foliares de B. coccolobifolia, $C$. calophyllum, $D$. mollis, $H$. stigonocarpa e S. lycocarpum de 500, 1240, 730, 2300 e $1330 \mu \mathrm{g} 100 \mathrm{~g}^{-1}$ e teores de licopeno em B. coccolobifolia, C. calophyllum e $S$. lycocarpum iguais a 270,120 e $20 \mu \mathrm{g} 100 \mathrm{~g}^{-1}$, respectivamente. Os autores não observaram teores de licopeno nos extratos foliares de $D$. mollis e $H$. stigonocarpa. 
Almeida et al. (2014) avaliaram duas espécies de Pereskia aculeata e Pereskia grandifolia, onde encontraram teores de $\beta$ caroteno de 24,070 e 6,210 $\mu \mathrm{g} 100 \mathrm{~g}^{-1}$ respectivamente. Já o teor de licopeno foi observado apenas na espécie Pereskia grandifolia igual a $6,440 \mu \mathrm{g} 100 \mathrm{~g}^{-1}$. O licopeno também funciona como um potente antioxidante devido ao grande número de ligações dieno conjugadas, absorvendo o oxigênio singleto (SILVA et al., 2010; BLUM et al., 2005).

Os teores de provitamina A, foram expressivos apresentando maior quantidade nos extratos foliares de $B$. coccolobifolia, $D$. mollis e Q. grandiflora respectivamente (Tabela 2). 0 menor teor foi encontrado para $S$. cernuum. Já Menezes Filho et al. (2018) verificaram quantidades inferiores às obtidos neste estudo para os extratos foliares de $C$. calophyllum, $D$. mollis, $H$ stigonocarpa e $S$. lycocarpum colhidas em diferente época do ano, (valores iguais a 0,1; 0,$1 ; 0,2$ e 0,1 RAE $100 \mathrm{~g}^{-1}$ respectivamente).

Os autores não encontraram a presença de provitamina A no extrato foliar de $B$. coccolobifolia, diferentemente deste estudo onde um teor considerável de provitamina $A$ foi detectado na espécie citada. Isso demonstra a necessidade de se estudar os extratos vegetais nas espécies do bioma Cerrado colhidas em diferentes estações do ano e diferentes fases fenológicas da planta, bem como se avaliar diferentes protocolos de extração, desde que estes fatores são causa de variação tanto quanto à presença como quanto à sua concentração na planta.

\section{CONCLUSÃO}

Os estudos realizados com extratos acetoetanólicos brutos nas dezessete espécies vegetais encontradas em um fragmento de Cerrado tipo cerradão, demonstraram a presença de expressivos conteúdos de compostos fenólicos totais e taxas de sequestro do radical DPPH, exceto nas espécies $A$. humile, $B$. intermedia e $K$. lathrophytum, as quais não apresentaram atividade antioxidante.

Os teores de $\beta$-caroteno bem como de provitamina A correlacionados, foram observados em apenas quatro espécies (B. coccolobifolia, $D$. mollis, $Q$. grandiflora e $S$. cernuum), e quantidades significativas de licopeno foram detectadas em todos os extratos foliares avaliados.
Os extratos vegetais das espécies do Cerrado apresentam uma ótima opção de uso para novas formulações para alimentos funcionais à base de produtos naturais com características químicas bioativas, bem como medicamentos farmacêuticos fitoterápicos manipulados para uso tópico e interno. Tanto os compostos fenólicos como os carotenóides e a provitamina A são substâncias envolvidas na prevenção de doenças ocasionadas por radicais livres, garantindo uma melhor qualidade de vida, além de que a utilização destas matérias primas proporciona a preservação das espécies vegetais tão importantes quimicamente.

\section{Agradecimentos}

Os autores agradecem ao Instituto Federal Goiano - Campus Rio Verde, GO, ao CNPQ, a CAPES, a FAPESP e a FAPEG pelas bolsas de mestrado (Antonio) e doutorado (Josemar e Marcela).

\section{Referências}

ALMEIDA, M. E. F.; JUNQUEIRA, A. M. B.; SIMÃO, A. A.; CORRÊA, A. D. Caracterização química das hortaliças não-convencionais conhecidas como ora-pro-nobis. Bioscience Journal, v.30, supplement 1, p. 431-439, 2014.

ANDRADE, C. A.; COSTA, C. K.; BORA, K.; MIGUEL, M. D.; MIGUEL, O. G.; KERBER, V. A. Determinação do conteúdo fenólico e avaliação da atividade antioxidante de Acacia podalyriifolia A. Cunn. ex G. Don, Leguminosae-mimosoideae. Revista Brasileira de Farmacognosia, v. 17, n. 2, p. 231-235, 2007. https://doi.org/10.1590/S0102$\underline{695 \times 2007000200017}$

BENDICH, A.; LANGSETH. L. Safety of vitamin A. American Journal of Clinical Nutrition, v. 49, n. 2, $\mathrm{p}$. 358-371, 1989.

https://doi.org/10.1093/ajcn/49.2.358

BLOIS, M. S. Antioxidant determinations by the use of a stable free radical. Nature, v. 1, n. 181, p. 199-200, 1958. https://doi.org/10.1038/1811199a0

BURQUE, R. K.; FRANCESCONI, L. P.; VICTORINO, A. T.; MASCARENHAS, M. Á.; CERESÉR, K. M. Determination of phenolic and evaluation of antioxidante activity of Lafoensia pacari (Lythraceae). Revista Eletrônica de Farmácia, 
v.12, n.1, p. $1-10, \quad 2015$. https://doi.org/10.5216/ref.v12i1.24645

BLUM, A.; MONIR, M.; WIRSANSKY, I.; BEM-AZIR, $S$. The beneficial Effects of tomatoes. European Journal of Internal Medicine, v.1, n.6, p. 402-404, 2005. https://doi.org/10.1016/j.ejim.2005.02.017

FERREIRA, E. S.; LUCIEN, V. G.; AMARAL, A. S.; SILVEIRA, C. S. Caracterização físico-química do fruto e do óleo extraído de tucumã (Astrocaryum vulgare Mart). Revista Alimentos e Nutrição, v. 19, n. 4, p. 427-433, 2008.

GALLON, M. E.; BARROS, B. S. P.; SILVA, M. A.; DIAS, S. H. M.; ALVES-DA-SILVA, G. Determinação dos parâmetros anatômicos, físico-químicos e fitoquímicos das folhas de Solanum lycocarpum A. St. - Hill. Revista Brasileira de Plantas Medicinais, v. 17, n. 4, supl. 2, p. 937-944, 2015. https://doi.org/10.1590/1983-084X/14 127

HALLIWELL, B.; AESCHBACH, R.; LOGIGER, J.; ARUOMA, $O$. I. The characterization of antioxidants. Food Chemistry Toxicology, v.33, n.7, p. 601-617, 1995. https://doi.org/10.1016/0278-6915(95)00024-V

LUIZ-FERREIRA, A.; COLA-MIRANDA, M.; BARBASTEFANO, V.; HIRUMA-LIMA, C. A.; VILEGAS, W.; BRITO, A. R. M. S. Should Anacardium humile St. Hill. Be used as na antiulcer agent? A scientific approach to the traditional knowledge. Fitoterapia, v.79, n.3, p. 207-209, 2008. https://doi.org/10.1016/j.fitote.2007.11.006

MALDINI, M.; MONTORO, P.; GIANGASPERO, A.; BALICK, M. J.; PIZZA, C.; DELLA LOGGIA, R. Screening of the tropical anti-inflammatory activity of the bark of Acacia cornigera Willdenow, Byrsonima crassifolia Kunth, Sweetia panamensis Yakovlev and the leaves of Sphagneticola trilobata Hitchcock. Journal Ethnopharmacology, v. 122, p. 430-433, 2009. https://doi.org/10.1016/i.jep.2009.02.002

MENEZES FILHO, A. C. P. de; OLIVEIRA FILHO, J. G de; CHRISTOFOLI, M.; SOUZA, C. F de. Atividade antioxidante, conteúdo de fenólicos totais, carotenóides e provitamina A em extratos vegetais do cerrado goiano. Revista Uniciências, v.22, n.1, p.28-32, 2018. https://doi.org/10.17921/1415-

5141.2018v22n1p28-32

MICHELIN, D. C.; SANNOMIYA, M.; FIGUEIREDO, M. E.; RINALDO, D.; SANTOS, L. C.; SOUZA-BRITO, A. R. M.; VILEGAS, W.; SALGADO, H. R. N. Antimicrobial activity of Byrsonima species (Malpighiaceae). Revista Brasileira de Farmacognosia, v. 18, p. 690-695, 2008. https://doi.org/10.1590/S0102$\underline{695 \times 2008000500009}$

NACZK, M.; SHAHIDI, F. Extraction and analysis of phenolics in food. Journal of Chromatography $A$, v.1054, n.1-2, p. 95-111, 2004. https://doi.org/10.1016/S0021-9673(04)01409-8

NAGATA, M.; YAMASHITA, I. Simple method for simultaneous determination of chlorophyll and carotenoids in tomato fruit. Nippon Shokuhin Kogyo Gakkaishi, v. 39, n. 10, p. 925-928, 1992. https://doi.org/10.3136/nskkk1962.39.925

NAIK, G. H.; PRYADARINIK, K.; SATAU, J. G.; BANAVALIKAR, M. M.; SOHONI, D. P.; BIYANI, M.; MOHAN, H. Comparative antioxidant activity of individual herbal componentes used in Ayurvedic medicine. Phytochemistry, v. 63, p. 97-104, 2003. https://doi.org/10.1016/S0031-9422(02)00754-9

NASCIMENTO, J. C.; LAGE, L. F. O.; CAMARGOS, C. R. D.; AMARAL, J. C.; COSTA, L. M.; SOUSA, A. N de.; OLIVEIRA, F. Q. Determinação da atividade antioxidante pelo método DPPH e doseamento de flavonóides totais em extratos de folhas de Bauhinia variega (L.). Revista Brasileira de Farmácia, v.92, n. 4, p. 327-332, 2011.

NUNES, F. R. S., DIAS, H. M. C., CAVALCANTE, G. $M$. Investigação das atividades antioxidante e antimicrobiana de duas espécies arbóreas ocorrentes no bioma Caatinga. Revista Estação Científica (UNIFAP), v. 6, n. 1, p. 81-90, 2016. https://doi.org/10.18468/estcien.2016v6n1.p81$\underline{90}$

PEREIRA, V. V.; BOREL, C. R.; SIVA, R. R. Phytochemical Screening, total phenolic content and antioxidant activity of Byrsonima species. Journal Natural Product Research, v.29, n.15, p. 1461-1465, 2015. https://doi.org/10.1080/14786419.2014.1002407 
PEREIRA, K. C. L.; De OLIVEIRA, A. K. M.; MATIAS, R.; RIZZI, E. S.; ROSA, A. C. Potencial alelopático do extrato etanólico de Anacardium humile A. St. -Hill. (cajuzinho-do-cerrado) na germinação e formação de plântulas de Lactuca sativa $L$. (Alface), Lycopersicon esculentum Mill. (tomate) e Senna obtusifolia (L.) Irwin \& Barneby (fedegoso). Gaia Scientia, v. 12, n. 2, p. 144-160, 2018.

SOARES, S. E. Ácidos fenólicos como antioxidantes. Revista Nutrição, v. 15, n. 1, p. 7181, 2002. https://doi.org/10.1590/S1415$\underline{52732002000100008}$

SOUSA, C. M. M.; SILVA, H. R. S.; VIIRA-JR, G. M.; AYRES, M. C. C.; COSTA, C. L. D. C.; ARAÚJO, D. S.; CAVALCANTE, L. C. D.; BRARROS, E. D. S.; ARAÚJO, P. B. M.; BRANDÃO, M. S.; CHAVES, M. $H$. Fenóis e atividade antioxidante de cinco plantas medicinais. Revista Química Nova, v.30, n.2, p. 351-355, 2007. https://doi.org/10.1590/S010040422007000200021

ROCHA, M. S.; FIGUEIREDO, R. W de.; ARAÚJO, M. A. M.; MOREIRA-ARAÚJO, R. S. R. Caracterização físico-química e atividade antioxidante (in vitro) de frutos do cerrado piauiense. Revista Brasileira de Fruticultura, v. 35, n. 4, p. 933-941, 2013. https://doi.org/10.1590/S0100-

\section{3}

RONCADA, M. J.; WILSON, D.; NUNES, M. F.; OKANI, E. T. Hipovitaminose A em filhos de migrantes nacionais em trânsito pela cidade de São Paulo, Brasil. Revista Saúde Pública, v. 12, p. 345-350, 1978. https://doi.org/10.1590/S0034$\underline{89101978000300009}$

SILVA, L. C.; COSTA, R. S.; SANTANA, A. dos S.; KOBLITZ, M. G. B. Compostos fenólicos, carotenóides e atividade antioxidante em produtos vegetais. Semina: Ciências Agrárias, v.31, n.3, p. 669-681, 2010. https://doi.org/10.5433/1679-

0359.2010v31n3p669

SILVA, R. A. O., SANTOS, M. R. M. C.; SANTOS FILHO, F. C.; SILVA, M. C. C.; OLIVEIRA, G. A. L.; SILVA, M. G. O.; MARQUES, L. G. A. Prospecção tecnológica de fitoterápico (Euphorbia tirucalli L.) utilizado no tratamento de neoplasias e outras doenças. Cadernos de Prospecção, v. 6, n. 4, p.
490-499, 2013. https://doi.org/10.9771/S.CPROSP.2013.006.052

SOUZA, W. A.; BOAS, O. M. G. C. V. A deficiência de vitamina A no Brasil: um panorama. Revista Panamericana de Salud Pública, v. 12, n. 3, p. 173-179, 2002. https://doi.org/10.1590/S102049892002000900005

VALKO, M.; IZAKOVIC, M.; MAZUR, M.; RHODES, C. J.; TELSER, J. Role of oxygen radicals in DNA damage and câncer incidence. Molecular and Cellular Biochemistry, v. 266, n. 1-2, p. 37-56, 2004.

https://doi.org/10.1023/B:MCBI.0000049134.691 $\underline{31.89}$

WU, J. H.; THUNG, Y. T.; WANG, S. Y.; SHYUR, L. F.; KUO, Y. H.; CHANG, S. T. Phenolic antioxidants from heartwood of Acacia confusa. Journal Agricultural Food Chemistry, v. 53, p. 5917-5921, 2005. https://doi.org/10.1021/jf050550m 